INTERNATIONAL DESIGN CONFERENCE - DESIGN 2018

https://doi.org/10.21278/idc.2018.0277

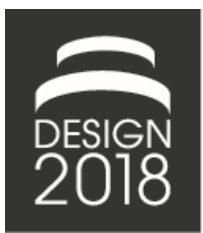

\title{
FRAMING STRATEGIC VALUE THROUGH DESIGN-LED INNOVATION PRACTICE
}

\author{
M. Bailey, N. Spencer, N. Smith, M. Aftab, C. Knott and P. Sams
}

\begin{abstract}
In university/industry collaborative projects, full project value is often overlooked and such projects can be viewed simply as contributing to student employability agendas. Initially, collaborators tend to place value solely on the projects' created outputs. This paper reveals how strategic value evolves during such projects and identifies frame creation as a means of highlighting additional values in design-led innovation projects. Identifying 'value-frames' allows the academic team to be more purposeful in aligning project focus to partner objectives, thereby increasing impact potential.
\end{abstract}

Keywords: value driven design, design education, innovation

\section{Introduction}

This paper is concerned with design-led innovation projects conducted by multidisciplinary postgraduate students working with external partner organisations and academics; and the values that such projects can deliver to those partners.

Designers are familiar with the notion of problem framing. Cross (2015) describes problem framing as 'generating perspectives that engender new understanding of the problem and offer bridges to new solutions'. Dorst (2015) presents Frame Creation, a detailed approach to innovation practice through framing. Furthermore, Dorst and Cross (2001) explain how problem and solution spaces (or frames) coevolve. They explain that the complex and networked nature of problems requires a bounded, or framed, exploration rather than solutionist approaches. Additionally, Mozota (2006) whilst highlighting the four powers of design i.e. design as a differentiator, design as an integrator, design as a transformer and design as good business concluded that each of these contribute towards creating substantial value for the organisation whilst framing problems and solutions.

This paper puts forward the case that the value(s) derived from such complex networked projects are framed and co-evolve as the project progresses. The research focuses on projects conducted as collaborations between academic staff specialising in design led innovation practices, Masters students from a Multidisciplinary Innovation programme, and middle and upper management employees from external partner organisations (hereafter referred to as the 'partner'). It puts forward the case for coevolving strategic value and presents the concept of 'Value Frames' as a means to examine and understand how organisations are using the outputs, knowledge and insights produced by design-led University collaborations.

This research is concerned with the question: How does value evolve during design-led project collaborations and what forms of strategic value emerge? This study examines three project collaborations. The research does not attempt to compare the projects with one another. Each project is a separate case illustrative of three distinct strategic Value Frames. The research seeks to understand how organisational value evolved during the collaborations, highlighting design-led practices that 
appear important in shaping and enabling that value. Each project, as a case study, demonstrates a shift in project targets and outputs. Each project was undertaken with the intent of delivering new product or service propositions, but in reality evolved, delivering different, additional forms of strategic value for the collaborating organisation.

In this study design-led innovation projects are run through a model of Integrated Academic Practice (IAP) (Bailey and Smith, 2016). IAP employs an enquiry-based pedagogy that involves students as coresearchers, engaged in research for, and with, the partner, working together with academics and professional practitioners. Using the project as the context for the enquiry, the process embeds both data-capture and testing to develop new knowledge about evolving approaches and practice(s). The IAP model works when stakeholders are embedded fully in the projects, working as co-creators within the team. Consequently opportunity exists for the individuals involved to learn through experience, allowing their ideas about the project to evolve as the project progresses.

\section{Literature review}

\subsection{Design-led innovation}

There is no conclusive agreement in literature regarding the precise role of design-led innovation nor its specific value (Design Council, 2016). However, there is concurrence regarding the importance of multistakeholder engagement. Bucolo and Matthews (2011) conclude that the main criteria for the success of their design-led innovation model is co-development, facilitated by design experts, with various stakeholders engaged throughout all stages of the design process. They suggest that the goal of designled innovation is to ground stakeholder conversations around future propositions. They also stress the role of design methods that allow for vivid visualisations of proposed solutions as an important tool in a design-led innovation model.

Norman and Verganti (2014) promote the involvement of external stakeholders, referred to as interpreters, in order to fuel disruptive innovation. The involvement of multiple stakeholders requires methods that engage in a purposeful way. Kembaren et al. (2014) suggest three key stages that lie behind the success of multi-stakeholder, design-driven innovation in organisations: 1, Sensing; 2, Sensemaking; 3, Specifying (setting up and storytelling as communication sub-stages). In the context of design-led social innovation, Manzini (2014) refers to a 'constellation of design initiatives' and multiple roles that designers can perform as both innovation triggers and facilitators of co-creative activity. $\mathrm{He}$ is consistent in reinforcing the importance of multi-stakeholder involvement and concludes that the designers' role is in 'making things happen' (ibid).

Whilst these authors consider approaches to achieving innovative output using a design-led approach, they do not stress the overall purpose of design, the value created by design or the values to each stakeholder of engaging in design-led innovation.

\subsection{Design value}

The value design has and how it can be applied within organisations has been explored within literature by numerous authors. Martin (2009) highlights how many organisations and leaders have turned to design methods to add value to their businesses, showing an openness to continuously redesign business practices. Yee et al. (2017) echo this through demonstrating seven roles of design that impact organisational transformation in order to achieve positive strategic change, citing the success of organisations including Deloitte, Spotify and Telstra. In addition, Cooper et al. (2016) found that organisations utilising design acquired benefits beyond styling, and describe how capabilities in design are amongst the five most important sources of competitive advantage alongside: the quality of products, quality of services, and relationship with clients, but ahead of capabilities in manufacturing.

When embedded within an organisation's culture and processes, design can deliver strategic value through providing an environment where iterative experimentation is an essential part of the design process (Kolko, 2015). Brown $(2009$, p. 32) emphasises the need for a culture ' $[\ldots]$ where people know they can experiment, take risks, and explore the full range of their faculties' in a safe environment where success is rewarded and failure is expected. In the case of the projects reviewed in this study, such an 
internal environment rarely exists for the partner employees involved. The university collaboration provides that safe environment for innovation (Bailey and Smith, 2010).

What is clear from the literature is that businesses in which design is applied, or granted permission to make decisions, do generate strategic value(s) by design. What the literature does not reveal is what strategic value(s) are derived from design-led multidisciplinary practices externally facilitated with enterprises where previously such designerly practices were absent, or of limited influence. The projects reviewed in this study are of this type.

\section{Methodology}

This study comprised three phases: The first phase reviewed 66 projects seeking to identify projects where the evolution of value was clear. This allowed the researchers to identify three projects where the evolution of value was clearly demonstrated and where the final organisational value was distinct and well articulated.

The second phase documented and interrogated the selected projects as case studies to describe the evolution of value and the final state of the value recognised by the partner.

The third phase sought to establish whether the types of value identified in the three case study projects were evident in the broader body of 66 projects reviewed. This final phase allowed the researchers to propose three distinct value frames.

\subsection{Phase 1}

All of the projects in the phase one review had, and delivered, a primary, intended, purpose - the development of a new product or service. Each project is documented with a:

- Project Brief, which contains the original project targets and expectations as well as a description of the project space;

- Project Materials produced during the project broadly catalogued under problem space, solution space, and strategic transition (understanding and plans to move the organisation to a position where it can deliver the solution propositions); and

- Project Value Interview (lead employees involved in the organisation are asked about the value they derive from the project and its potential impact on the organisation).

This review identified three areas where strategic value, beyond the brief, appeared to emerge:

1. development of new strategic direction,

2. changes in working practice or attitude and

3. designed assets used to leverage strategic investment.

\subsection{Case study review}

Phase two focused on three projects that delivered unexpected, additional value that was well documented and aligned with one of the three values identified in phase one. In each case it was clear that that the project had evolved to produce outputs and outcomes beyond new product and service propositions and that these were leveraged within the partner organisation to different strategic effect. Each of these projects was formed as a case study and analysed in order to describe to determine and describe:

- Collaborator Context (to understand the problem setting);

- Original Project Challenge (to understand the questions, priorities and targets of the work)

- Key Activities (to describe the design-led approach influencing the evolution of value)

- Actions (a summary of key standpoints, insights and positions (frames))

- Key Finding

- Project Outputs

- Project Value

For each case study the researchers sought to understand how the project actions influenced the evolution of value. They also sought to understand: the organisational targets the work related to and if the work produced suggested new targets; if the work encouraged entrepreneurial practices and creative 
confidence within the organisation and; if the work had a direct impact on funding and resource allocation decisions. Semi-structured, independent project value interviews, conducted with key partner employees, informed this understanding.

\subsection{Value mapping}

Having, through phase two, understood more clearly, the nature of the three apparent types of value, the 66 projects were mapped against these three values in order to see whether there was any pattern and whether this would enable the researchers to frame the types of value. What emerged was a picture that illustrated that projects fell broadly into one of the three categories of value observed in Phase 1 and typified by the three case studies. Some projects sat very clearly in just one category whilst others straddled two or all three. The volume or intensity of value also varied. However, their general grouping did allow the researchers to draw some conclusions regarding the definition of the three emerging value frames delivered by this type of multidisciplinary design-led university/industry partnership project (Figure 1):

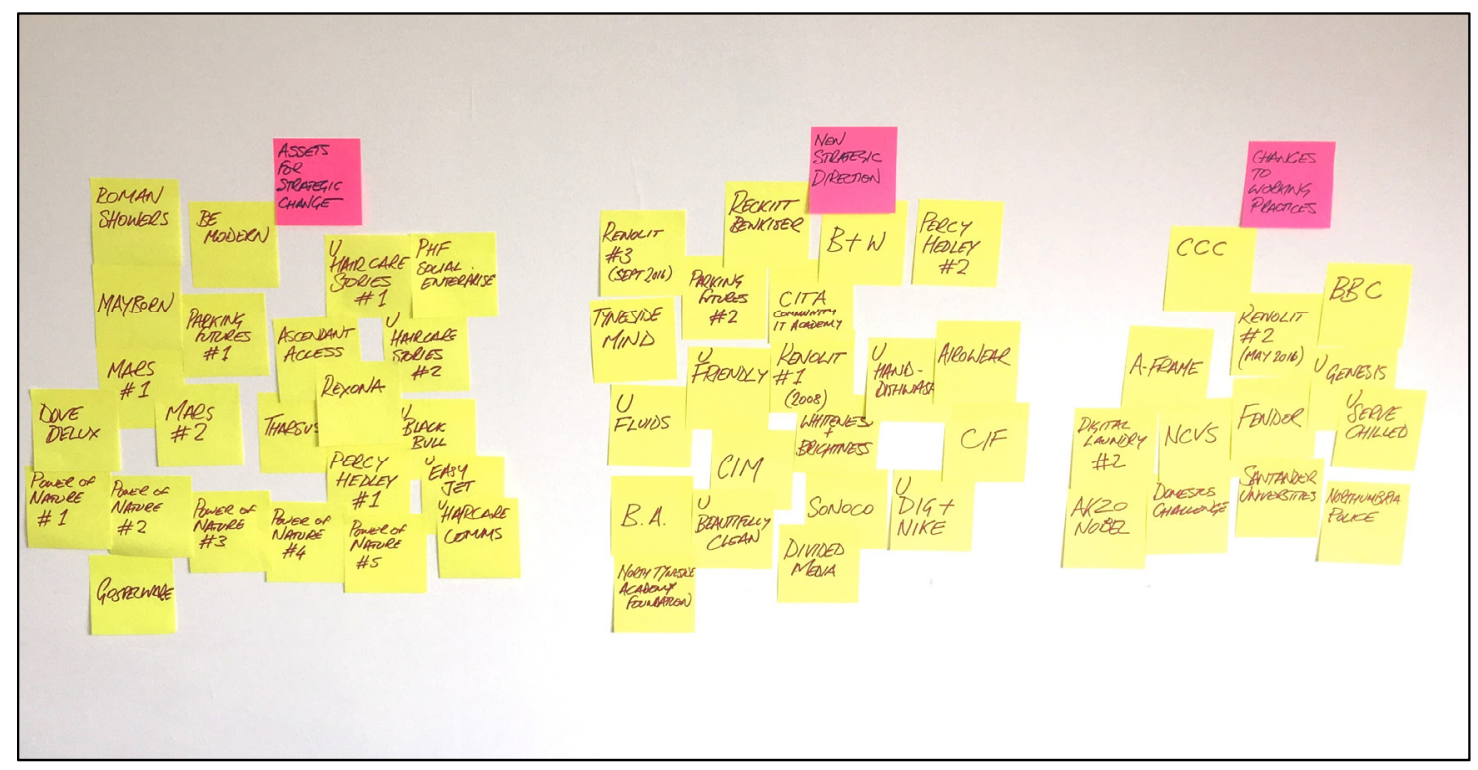

Figure 1. Projects clustered by strategic value

\section{Case studies}

Each project case study, presented in this section, involved the following common actors and roles:

- Senior academics (Design, Business \& Technology) $\mid$ project framing, direction setting, critical evaluation

- Multidisciplinary Innovation Master's students $\mid$ mixed research methods, mixed design and business methods, co-creative practice

- Practice-based Researchers | literature and contextual practice review, solution development, communication \& trialling

- Key partner stakeholders | Co-creators of understanding, framing, and solution trialling.

Dorst provides valuable guidance for frame creation. He defines 9 steps. In the projects reviewed in this research, there are 4 primary actions that map, broadly, onto the 9 steps: 'Find Out' (understand and illustrate the complex challenge situation - what is it as a system, including processes, materiality and people; how different people view the situation and to what, within the system, their perspective relates); 'Form Positions' (illustrate what the situation is and could be like); 'Explore Solutions' (materialise through motion, shape and form); 'Communicate Narratives' (create engagement materials) (Table 1). 
Table 1. Frame Creation steps relative to Project Actions

\begin{tabular}{|l|l|}
\hline Dorst's Frame Creation steps & \multirow{2}{*}{ Project Actions } \\
\hline Archaeology & Find Out \\
\cline { 1 - 1 } Paradox & \\
\hline Context & \multirow{2}{*}{ Form Position } \\
\hline Field & \multirow{2}{*}{ Explore Solutions } \\
\hline Themes & \\
\hline Frames & Communicate Narratives \\
\hline Futures &
\end{tabular}

\subsection{Case study 1: Percy Hedley Foundation}

Collaborator context: The Percy Hedley Foundation $(\mathrm{PH})$ is a charity providing specialist, high quality, and innovative services for children, young people and adults with a range of physical and social needs. PH, within their Adult Services function, have developed a range of service-user enterprise ventures: Orion - professional print service, website development and music production; and Craftworks - producing and selling a range of wooden, card, and horticulture craft products. These have emerged as a consequence of opportunity and staff interest. The organisation felt that now was an appropriate time to consider Social Enterprise as a potential revenue stream and to consider more broadly the appetite for developing the role of enterprise within the Foundation. The key stakeholders involved in this project were 50 members of $\mathrm{PH}$ (trustees, leadership team, management, care staff and service-users).

Original project challenge: The original project challenge set by the partner was to explore what new enterprises could be established within the context of the organisation and service user capabilities.

Key activities - Through site visits, semi-structured interviews with members of the leadership and governance team, and discussions with a range of staff and service users the team developed an understanding of what 'enterprise' means and could mean to PH. Literature and regional best practice case studies were used to situate understanding emerging from within $\mathrm{PH}$ about enterprise. These research activities took place concurrently with design-led innovation practice feeding and being informed by on-going discussions between the academic lead and the Foundation's Director of Adult Services. 16 Multidisciplinary Innovation masters students' design-led innovation practice explored how new or modified services could be materialised through a culture of enterprise and how they may be developed, run and evaluated. During this project the students designed, developed and ran two co-creative workshops with a cross-section of $\mathrm{PH}$, including: trustees and members of the executive team; management and support staff from the school, adult services and residential care; and dayservice users. Their practice drew together a network of stakeholders with different priorities and perspectives to:

- Collaboratively develop a common understanding of 'enterprise' and 'enterprising'

- Explore how this understanding might materialise within the organisation,

- Consider what consequences, rights and responsibilities, might result.

Understanding and thinking emerging from across this range of activity was integrated into a set of principles and practices which formed the recommended 'model of enterprise'.

Actions:

1. Find Out - What does enterprise mean within $\mathrm{PH}$ and why does it hold value?

2. Form Position - What would it look and feel like if PH had an enterprising culture and coordinated approach to enterprise?

3. Explore Solutions: Development of a program of project-focused enterprising endeavours, which united staff and service users to use new and existing day services to plan, promote, produce and disseminate (exhibit or trade). 
4. Communicate Narratives: Creation of a number of narratives and materials illustrating this programme and the development the short, medium and long term needs of the organisation.

Key findings in the project - PH want to develop and show off the talents of their service users; they want to be known as a leading enterprising charity; they are worried about the impact that being business focused will have on care (or perceptions of care); $\mathrm{PH}$ are very enterprising but have yet to coordinate their approach, development and delivery.

Project output - A model of enterprise (purpose, roles, output and impact) for the whole organisation and support resources, locating a system of planning, delivery and evaluation against the organisation's strategic objectives.

\subsection{Case study 2: Good Careers Guidance}

Collaborator context - Churchill Community College (CCC) is one of 13 schools and 3 colleges participating in the Gatsby Good Careers Guidance pilot project (NELEP, 2015), which is managed by the North East Local Enterprise Partnership (NELEP). The pilot aims to test the eight benchmarks identified in the Good Careers Guidance report (Holman, 2014) and collect evidence on the impact of structuring career information, advice and education guidance in this way. In January 2016, as part of the pilot, CCC successfully secured funding allowing Northumbria University to undertake designled research to produce understanding and solutions to help address the Gatsby Good Careers Guidance Benchmark 6: Experiences of the Workplace: 'Every student should have first-hand experiences of the workplace through work visits, work shadowing and/or work experience to help their exploration of career opportunities, and expand their networks' (Gatsby Good Careers Guidance Report).

Gatsby refers to 'Experiences of the Workplace' as opposed to work experience that has a tradition and history that can be limiting when thinking about how schools might respond to and address this benchmark. Experience of the workplace does not have to involve the traditional one or two-week placement; effective experience programmes can involve, but are not limited to: work shadowing, 'take your son or daughter to work' days, extended school visits to workplaces and episodic work experience over a longer time period, interspersed with periods in school. The key stakeholders involved comprised staff and pupils of CCC, staff from schools within the pilot scheme, careers guidance experts and members of the NELEP.

Original project challenge - Produce implementable solutions to help CCC address the Gatsby Good Careers Guidance Benchmark 6: 'Experiences of the Workplace'. Solutions must be sustainable beyond the Pilot Project funding and must be transferable to other schools to enable national scaling.

Key activities - Conducting desk research and interviews with key staff from the various institutions involved in the project, as well as running co-creative workshops with pupils and participants from across the organisation (Figure 2). Delivering key insights and clear positions to respond to Benchmark 6.

Actions:

1. Find Out - What is a 'meaningful' experience of the workplace and how can different schools within different contexts deliver this?

2. Form Position - Due to contextual differences, different schools deliver careers guidance in very different ways meaning that solutions would need to be flexible and adaptable.

3. Explore Solutions - Individual concepts were developed and trialled before a three-stage framework was designed. Designed templates and resources supported the framework.

4. Communicate Narratives - Deploying the resources in 6 pilot schools identified a need to redesign the resources to adopt schools' vernacular in order to be more readily accepted and used. This also generated 6 case studies of successful application of the framework. 


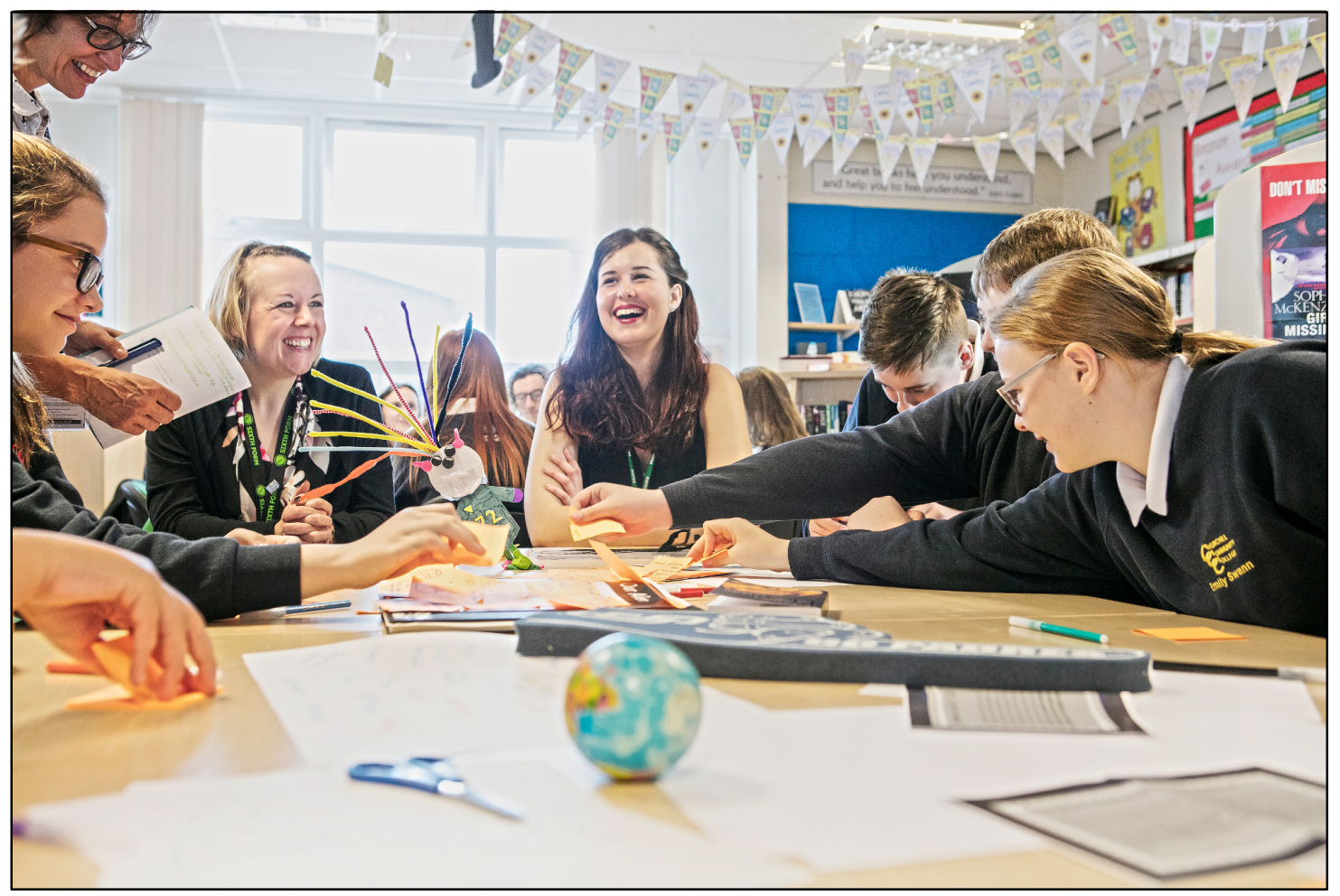

Figure 2. Students facilitating co-creative workshop with academics, researchers, pupils and staff of $\mathrm{CCC}$

Key findings in the project - Pupils who did identify a chosen career or industry had limited knowledge of access pathways; pupils tended to have a singular focus rather than being open to flexibility and change in their future career.

Project output - A Careers Guidance Framework designed to ensure a holistic approach to careers guidance in schools. This framework empowers teachers to develop a programme of activities that is innovative and enterprising and delivers meaningful careers guidance as well as providing a structure for schools, businesses and 3rd party providers to use when delivering careers guidance. Templates and guides have been provided alongside evidential case studies for reference.

\subsection{Case study 3: Power of Nature}

Collaborator context: The collaborating partner is a global Fast Moving Consumer Goods manufacturer exploring highly disruptive new science and technology innovation for a range of household applications. Adoption of the new technology has the potential to disrupt the business and sector. 18 Multidisciplinary Innovation students were involved. Key stakeholders were 2 research scientists ( 1 the partner's project lead, 1 a Visiting Professor 'science interpreter'), typical consumers.

Original project challenge: The principal challenge was to translate complex, new and in some cases negatively perceived by the general public, scientific discoveries into meaningful and compelling product concepts and communication material in order to generate internal business support for continued research.

Key activities - Detailed briefings with the partner's lead project scientist set the macro context (global mega-trends, policy context, business context) and the micro context (the science itself, how it works, what it could do and how this might relate to individual consumers in specific instances); extensive interpretation sessions with the Visiting Professor using analogies, metaphors and storytelling as an approach to: a, learn about the science in layman's terms and b, explore possible design-communication directions; desk-research; observational research of user behaviour in potential application scenarios; design conceptualisation; business-model conceptualisation; iterative development and refinement of communication strategies and associated collateral. 


\section{Actions:}

1. Find Out - Understand the science, understand potential consumer benefit(s) and supporting business cases

2. Form Position - Based, in-part on the Macro-Micro toggling approach (Bailey et al. 2016), the team identified that in order to gain traction and therefore strategic buy-in, impactful communication would need to focus on end-user (micro) benefit (translating to new business opportunities) rather than corporate or environmental (macro) worth which is ultimately the desired and intended consequence of commercial success.

3. Explore Solutions - Through adopting speculative design approaches, multiple alternative communication concepts in early mock-up form (animations, faux-adverts (both film and poster), storyboards, product mock-ups and prototypes) were rapidly developed and shared with the partner and potential consumers. This was an iterative approach with multiple cycles leading to refined concepts.

4. Communicate Narratives - For internal (client organisation) communications a suite of macro (corporate, environmental) assets were envisioned, supported by micro (consumer benefit) material. In this way, the lead research scientist was able to reach beyond the scientific community in order to explain the full business potential, in consumer-facing terms that sales, marketing, commercial functions within the business would understand. The narrative was presented in 'their language' through highly professional, believable, finished materials.

Key findings in the project - The most complex (scientific) concepts can be broken down into simplistic explanations using metaphor and storytelling as part of a briefing process with a design-led multidisciplinary innovation team. This has a number of benefits: it reveals where misunderstanding amongst lay-people might lie; it necessitates the creation and adoption of new forms of description which may, in turn, lead to concepts for the final delivery material; it can identify new potential market opportunities as descriptions are explored and tested; identifying key audiences and adopting their language is a key aspect of position forming.

Project Output - A portfolio of communication pieces each of which presents specific complex science deployed to deliver end-user benefit in different use scenarios and global markets. These have been created to suit both internal and external audiences in a way that brings the science to life through speculative design that recognises the multiple different perspectives from which different stakeholders would draw benefit and value.

\section{Research findings: Three Value-Frames}

Strategic Value, 'the degree to which a particular action or planned action is important or useful in relation to something that [the enterprise] wants to achieve' (Cambridge Dictionary, 2018), can evolve through university/industry collaborations as an added value beyond the new product or service that was the primary purpose of the collaboration. Furthermore, such values can be framed through the four actions: Find Out; Form Position; Explore Solution and Communicate Narratives. The three Value Frames identified in this study are:

\subsection{Co-created strategy}

Co-created strategy is the identification of a new business target and plan for achieving it. It takes advantage of designerly activities in order to support strategic organisational review and direction setting.

Designerly activities allow participants to 'see' future potential by adopting alternative frames through which to view their business activities. These may include the production of visions of new products or services or new enabling collateral. Such collateral delivers value by encouraging partners to shift their mind-set by taking the available data about a given situation and framing it in order to present alternative meanings derived from it. Whilst the tangible outputs of projects delivering value within the co-created strategy frame may be proposals, artefacts and plans, the true value is in the intellectual transformational mind-set of reframing. 
For example, for Percy Hedley, the project value was a new way of understanding Enterprise and its potential value to the Foundation. The academics leading the work formed the position to support the partner's agenda by considering the question: how can the principles of enterprise be applied to generate greater self-development and social inclusion opportunities for those who currently utilise day-services and have a range of impairments, and what organisational adaptations would be required? PH now use enterprise as a mechanism. Through this mechanism people at Percy Hedley are engaged in a programme of project-focused endeavours, consisting of day-to-day activities that are purposeful for the endeavour and meaningful for the individual. This understanding is now written into their development strategy and represents a significant shift in organisational mind-set.

In this case we can see the key importance of co-creative activity with a wide range of stakeholders. This was key to changing the perception of care, which in turn, unlocked a new way of envisioning the enabling qualities of enterprise as a dimension of care-giving. The co-creative nature of the work that led to this realisation made it possible to shift organisation mind-set with regard to developing and introducing an enterprise strategy. By deploying designed collateral based on co-creative activity with the stakeholders the project established a collective sense of ownership in the future direction.

Engagement in, and outputs of, the project resulted in the partner understanding their challenge in a different way or from a different perspective. As a result of working co-creatively with the design-led team, enterprises are enabled to re-frame their proposition and envision alternative strategies.

\subsection{Creative Functionality}

Creative Functionality shifts the innovation mind-set of the organisation by helping enterprises to adopt a more entrepreneurial attitude to innovation through capitalising on latent internal capabilities and capacity.

It uses design-led approaches to develop or adapt organisational functionality (systems, processes, and behavioural practices). For those individuals involved it grants permission to adopt a more entrepreneurial attitude to innovation and experimentation. Through design-led, multidisciplinary cocreative activities, which take advantage of the context of the organisation, value can be delivered to the partner through frameworks, processes and organisation-specific tools. These promote bounded, or 'safe', creative risk-taking to be established within organisational culture.

For example, CCC and NELEP now have an evidence-based framework, co-developed with the university multidisciplinary team, with which to drive a 'safe' creative approach to developing contextrelevant solutions and creative culture-change in schools. This is very different from the declared 'actionable solutions' required of the initial brief, but offers a sustainable means by which schools can create and deploy their own solutions.

This was a highly complex project with multiple stakeholders possessing conflicting objectives, but an overriding desire to receive implementable solutions. By recognising the wide variation in contexts within which the solutions were required, the academics were able to form the position that an alternative approach was required. This led to the team co-creating a framework that would enable stakeholders in each context to develop their own solutions. Acceptance of this approach was only truly achieved when its effectiveness was demonstrated, and repeated, in real-world settings. This suggests that emphasis should be placed on establishing means of early demonstration within Creative Functionality in the future in order to achieve earlier buy-in.

\subsection{Leveraging Strategic Change}

Leveraging Strategic Change uses design-led approaches to help the partner visualise the future impact of innovations and thereby drive organisational strategy, funding and resources. It is achieved through the development of critical insights and explorative ideas delivered through compelling narratives presented as a range of designed collateral (e.g. adcepts, posters, artefacts, videos, campaigns etc.). All of these have the purpose of bringing ideas to life in tangible ways that are presented in appropriate language for any given decision-making audience thereby allowing innovation opportunities to be evaluated from different disciplinary and functional perspectives.

For example, Power of Nature started as an experiment to see whether the design-led approach would produce outputs that could influence internal decision-makers. Key to the success of this work was the 
position formed by the academics, and partner, that multiple audiences required multiple communication strategies capitalising on understanding of the language of each audience. An on-going developing portfolio of associated projects with the same partner is evidence that the design-led speculative outputs of these projects (user-focused design concepts employing the new science and delivered as highly professional marketing material simulations) are strong tools that assist the partner in leveraging strategic internal, external-collaborator, trade-body and research council backing to bring the technology to market.

Leveraging Strategic Change is about enablement. The case study research showed revealed a critical interpretive role required in order to ensure the materialised narratives are presented in the right language for the intended audience thereby delivering enablement. In this case, a highly design literate interpreter (the Visiting Professor), coupled with deep knowledge of the partner's various functional traits which the academic team had built up over years of collaboration, fulfilled this role. Recognising the fundamental importance of this in delivering outputs that leverage strategic change, in future IAP projects, emphasis can be placed on its importance in the project setup and recruitment of actors.

\section{Learnings and further research}

It is evident that a single project may deliver value across all three value-frames and that there are multiple factors that will determine whether the potential value as identified at a project's conclusion can be realised in the longer-term. Understanding and being able to influence the factors involved in delivering strategic value over the long-term is an important next step in ensuring that these types of design-led interventions deliver the desired impact as well as value.

Through the use of design, it is well understood and accepted that problem and solution understanding co-evolve while solving complex, ill-structured, wicked problems (Dorst and Cross, 2001). Good Careers Guidance is a good example of this. Through design-led innovation practice, whilst attempting to develop solutions, the team also developed in-depth understanding of the problem. This evolved understanding did not necessarily lead to a refinement of the solutions being developed but led to a new way of seeing that, in turn, led to describing new ways of doing. It shifted the frame through which the challenges and constraints could best be resolved and from which solutions could be developed.

In reviewing the four actions across the three case studies, it is evident that value creation is most impacted by Form Position and Communicate Narratives. Find Out (research) and Explore Solutions (design and develop) are essentially 'standard R\&D' practices.

Considering the 7 roles of design identified by Yee et al. (2017) in commercial design practice: Cultural Catalyst; Framework Maker; Humaniser; Power Broker; Friendly Challenger; Technology Enabler; Community Builder, in relation to these university/industry multidisciplinary design-led project value frames it is possible identify an $8^{\text {th }}$ role: Expert Authority. This is the role performed, predominantly by the academics, but informed by the students, researchers, partner and stakeholders, when they Form Position.

This study has allowed a conceptual framework to be established that defines three distinct value-frames but it poses further questions:

- Is it possible to use this knowledge to be more purposeful in attempting to set-up projects to deliver specific strategic value?

- What is the long-term value: without further intervention, how sustained and prevalent will the value be within the partner organisations?

\section{Conclusion}

This review has shown that strategic value in design-led multidisciplinary university/industry projects co-evolves alongside problem understanding and solutions development. Understanding that this is most greatly influenced by position forming means that it should be possible to be more explicit about what value(s) a project is intended to deliver at its inception and focus on ensuring that these are delivered. This means that the academic team, in framing and establishing the project with a partner will be able to work with them to form a collaborative position for the project focussing on the desired strategic goal whilst being cognisant of other emerging strategic values may emerge as the project progresses. 
Within an educational setting, having the ability to be explicit about these value-frames and the different perspectives that they offer, allows students understanding of the multiplicity, impact and value that design-led innovation offers organisations. Consequently this means that a balanced curriculum can be planned through which examples of each value-frame are experienced.

What this review has clearly shown is that, whilst the activities, resources and actions deployed in these projects are essentially the same, there are values created (beyond the student outputs) from which partners who are deeply engaged in the projects will benefit. Beyond the value delivered by responses to the original brief, these values offer the potential to deliver impact within both the organisational setting and academe.

\section{Acknowledgments}

The authors would like to acknowledge the support and creative energy of the Multidisciplinary Innovation Masters students who have conducted the projects reviewed in this research. They would also like to acknowledge the hard work and commitment on the Innovators in Residence and Research Associates involved. Particular gratitude is owed to the staff and service users of the Percy Hedley Foundation; the staff and pupils involved from Churchill Community College and North East Local Enterprise Partnership; and the scientists and stakeholders from the global FMCG company - you know who you are!

\section{References}

Bailey, M. and Smith, N. (2010), "Safe Environments for Innovation-Developing a New Multidisciplinary Masters Programme", Proceedings of EPDE 2010 / the 12th International Conference on Engineering and Product Design Education - When Design Education and Design Research meet..., Trondheim, Norway, September 23, 2010, The Design Society, Glasgow, pp. 60-65.

Bailey, M. and Smith, N. (2016), "Making it Work; Integrated Academic Practice", Proceedings of the 20th DMI: Academic Design Management Conference Inflection Point: Design Research Meets Design Practice, Boston, USA, July 28-29, 2016, DMI, Boston, pp. 2346-2363.

Bailey, M., Spencer, N. and Sams, P. (2016), "What on Earth is Responsible Innovation Anyway? - making it happen", Proceedings of the EPDE 2016 / 18th International conference on Engineering and Product Design Education, Aalborg, Denmark, September 8-9, 2016, The Design Society, Glasgow, pp. 234-239.

Brown, T. (2009), Change by design: how design thinking transforms organizations and inspires innovation, Harper Business, New York.

Bucolo, S. and Matthews, J.H. (2011), "Design led innovation: Exploring the synthesis of needs, technologies and business models", Proceedings of Participatory Interaction Conference 2011 (PINC 2011), Sønderborg, Denmark, January 13-15, 2011, University of Southern Denmark, pp. 354-357.

Cambridge Dictionary (2018), Strategic value. [online] Available at: https://dictionary.cambridge.org/dictionary/english/strategic-value (accessed 05.03.2018).

Cooper, R., Hernandez, R., Murphy, E. and Tether, B. (2016), Design value: The role of design in innovation, Lancaster University, Lancaster.

Cross, N. (2015), Endorsement of "Frame innovation: Create new thinking by design" by Dorst, K. [online] MIT Press. Available at: https://mitpress.mit.edu/books/frame-innovation

Design Council (2016), Invitation to Tender. [online] The Design Council. Available at: http://www.designcouncil.org.uk/sites/default/files/asset/document/Designled\%20innovation\%20ITT\%2004.11.16\%20(2).pdf (accessed 01.03.2017).

Dorst, K. (2015), Frame innovation: Create new thinking by design, MIT Press, Massachusetts.

Dorst, K. and Cross, N. (2001), "Creativity in the design process: co-evolution of problem solution", Design Studies, Vol. 22 No. 5, pp. 425-437. https://doi.org/10.1016/S0142-694X(01)00009-6

Holman, J. (2014), Good Careers Guidance. [online] The Gatsby Foundation. Available at: http://www.gatsby.org.uk/uploads/education/reports/pdf/gatsby-sir-john-holman-good-career-guidance2014.pdf (accessed 01.04.2016).

Kembaren, P., Simatupang, T.M., Larso, D. and Wiyancoko, D. (2014), "Design Driven Innovation Practices in Design-preneur led Creative Industry”, Journal of technology management \& innovation, Vol. 9 No. 3, pp. 91105. https://doi.org/10.4067/S0718-27242014000300007

Kolko, J. (2015), "Design thinking comes of age”, Harvard Business Review, Vol. 93 No. 9, pp. 66-71.

Manzini, E. (2014), "Making things happen: Social innovation and design”, Design Issues, Vol. 30 No. 1, pp. 57 -66. https://doi.org/10.1162/DESI_a_00248

Martin, R.L. (2009), The design of business: why design thinking is the next competitive advantage, Harvard Business Press, Boston. 
NELEP (2015), Good Careers Guidance Benchmarks Pilot. [online] North East Local Enterprise Partnership. Available at: https://www.nelep.co.uk/skills/north-east-ambition/ (accessed 01.12.2017).

Norman, D.A. and Verganti, R. (2014), "Incremental and radical innovation: Design research vs. technology and meaning change”, Design issues, Vol. 30 No. 1, pp. 78-96. https://doi.org/10.1162/DESI_a_00250

Yee, J., Jefferies, E. and Michlewski, K. (2017), Transformations: 7 Roles to Drive Change by Design, BIS Publishers, Amsterdam.

Mark Bailey, Director of Innovation Design and Learning \& Teaching

Northumbria University, Design

Northern Design Centre, NE8 3DF Gateshead, United Kingdom

Email: mark.bailey@unn.ac.uk 